\title{
Light scattering at the liquid to glass transition
}

\author{
M. Fuchs and A. Latz \\ Physik Department, Technische Universität München, D-8046 Garching, Germany
}

(Received 4 February 1991; accepted 29 July 1991)

\begin{abstract}
Light scattering in a viscoelastic liquid is studied. The change from liquid to solid-like Brillouin spectra is explained using frequency dependent elasto-optic constants. Within a standard dielectric model microscopic formulas for these generalized frequency dependent constants and the spectra are obtained. The phenomenological pictures of light scattering in an amorphous solid and in a liquid are obtained as high and low frequency limits. The observation of a Rytov Dip in a system of optically isotropic particles is predicted for slow enough viscoelastic relaxation. Approximations using the Mode Coupling Theory allow the spectra to be expressed in terms of density fluctuations alone. The predictions of this theory for the spectra are discussed.
\end{abstract}

\section{INTRODUCTION}

Light scattering can be used to obtain information about the static structure and the dynamics of gases, crystals, liquids, and amorphous solids (glasses). ${ }^{1}$ The spectra from liquids change drastically but continuously to the ones of glasses upon supercooling the liquid. ${ }^{2,3}$ Polarized spectra mainly result from density fluctuations leading to the observation of the dynamic structure factor. Macroscopically density fluctuations are coupled to dielectric fluctuations via the thermodynamic derivative $(\partial \epsilon / \partial n)_{T}{ }^{4}$ Depolarized spectra are differently described for different systems. In a crystalline or amorphous solid the dielectric fluctuations are coupled to the strain tensor. ${ }^{5}$ Depolarized spectra of molecular liquids are explained with the anisotropy of the molecular polarizability tensor. ${ }^{6,7}$ In gases and simple liquids of optically isotropic particles multiple scattering leads to depolarization. ${ }^{8}$

The fitting together of the phenomenological pictures for depolarized light scattering at the liquid to glass transition presents difficulties for two reasons: (1) In the solid a continuum description is used to explain the observation of transverse phonon peaks. In the liquid however the molecular anisotropy of the polarizability tensor is needed for describing depolarized spectra. The connection of the phenomenological continuum description in the solid and the microscopic ansatz for the liquid is not clear at the glass transition. ${ }^{9}$ The similarity of the underlying equilibrium structure of a glass and a supercooled melt indicates, that a microscopic model should reduce to the macroscopic pictures above and below the transition. (2) It is a well known fact of glassy dynamics ${ }^{10}$ that frequency dependent generalizations of transport coefficients and thermodynamic derivatives are observed. For example the dynamic specific heat $c_{p}(\omega)$ can be measured by heat spectroscopy. ${ }^{11}$ In the same way the elasto-optic, Pockels constants have to be generalized to frequency dependent functions. Rytov ${ }^{12}$ explored this frequency dependence within a phenomenological continuum model.

In this paper starting from an established dielectric model for a system of isotropic point particles ${ }^{13}$ a microscopic picture for light scattering in supercooled liquids is developed.The drastic qualitative changes of Brillouin scattering spectra from liquid to solid-like behavior are explained. The phenomenological pictures of light scattering in an amorphous solid and of scattering by density fluctuations in a liquid are obtained as two limit cases. Microscopic expressions for generalized frequency dependent elasto-optic constants are derived. The observation of a Rytov Dip ${ }^{14}$ in the depolarized spectrum is identified as a feature of the frequency dependence of these elasto-optic constants. This negative intensity, which is cut out of the central line in the depolarized spectrum, is generally explained by the coupling of the molecular anisotropy density and the transverse current. ${ }^{1}$ In this paper it is shown, that even for point particles with scalar polarizability the frequency dependence of the Pockels constants leads to a Rytov Dip. For experimental observation the viscoelastic relaxation has only to be slow enough. The microscopic formulas can be approximated in terms of the wave vector dependent dynamic structure factors using the Mode Coupling Theory. Asymptotic formulas of this theory allow to calculate the light scattering spectra at the liquid to glass transition. In a mesoscopic frequency range simple laws are obtained.

The dielectric model used here leads to the inclusion of multiple scattering from density fluctuations. This process has successfully been used to explain spectra from gases ${ }^{15,8}$ and simple liquids like liquid argon. ${ }^{16}$ The necessity of taking it into account in supercooled liquids becomes clear from the following physical picture: ${ }^{16}$ In a low viscosity liquid or gas the molecules are instantaneously isotropically disposed around a central one. On the time scale $1 / \omega$, where $\omega$ is the frequency shift, the molecules have already relaxed to their isotropically scattered equilibrium positions. When the structural relaxation time $\tau$, which gives the lifetime of an anisotropic distribution of molecules around a central one, increases to $\omega \tau \approx 1$ however, multiple scattering does not average away and contributes depolarized intensity. It is clear, that this picture applies to a liquid as well as to an amorphous solid, where the distribution of molecules has frozen out to form a metastable equilibrium state.

The contents of this paper are arranged as follows: Sec. 
II presents dielectric preliminaries. The phenomenological coupling of dielectric fluctuations to the strain and to density fluctuations is introduced. Results of a microscopic dielectric model are summarized. In Sec. III new microscopic formula for the spectra and elasto-optic constants are derived; these are discussed in Sec. IV, especially the limits of equilibrium liquid and stable glass are obtained. Section $V$ approximates the exact expressions in terms of density correlators. Spectra are calculated using results of the Mode Coupling Theory of the liquid to glass transition.

\section{DIELECTRIC MODEL}

Brillouin or dynamic light scattering ${ }^{1,5}$ measures fluctuations of a local dielectric function $\epsilon_{i j}$. In an homogeneous, isotropic and infinite system its average reduces to the scalar dielectric constant $\left\langle\epsilon_{i j}\right\rangle=\epsilon_{0} \delta_{i j}$. Introduction of a spatially averaged local dielectric function is possible, because the wave vector transfer $\mathbf{q}$ between incoming and scattered photon is small compared to the reciprocal interatomic distance $a: q a \ll 1 .^{19}$

For an incoming photon with frequency $\omega_{0}$ and polarization $\lambda=H, V$ (horizontal or vertical with respect to the scattering plane) the intensity of scattered photons with polarization $\lambda^{\prime}$ is given by ${ }^{1}$

$I^{\lambda \lambda^{\prime}}=\frac{I_{0}^{\lambda}}{R^{2}}\left(\frac{\omega_{0}}{c}\right)^{4} \frac{1}{16 \pi^{2}} \frac{k T}{2 \pi} \int d t e^{i \omega t}\left(\epsilon_{\lambda \lambda^{\prime}}(\mathbf{q}, t) \mid \epsilon_{\lambda \lambda^{\prime}}(\mathbf{q})\right)$.

The wave vector transfer obeys the Bragg condition $q=2 n_{r} k \sin (\theta / 2)$. The frequency shift equals $\omega=\omega_{0}-\omega_{s c}$. The Kubo scalar product $(A \mid B)$ $=(1 / k T)\left\langle\delta A^{*} \delta B\right\rangle$ is used. Dropping the prefactor in Eq. (1) the spectrum runs $\bar{I}^{\lambda \lambda^{\prime}}(q, \omega)=\hat{e}_{i}^{\lambda} \hat{e}_{j}^{\lambda^{\prime}}\left(\epsilon_{i j}(\mathbf{q}, \omega) \mid \epsilon_{k l}(\mathbf{q})\right)$ $\hat{e}_{k}^{\lambda} \hat{e}_{l}^{\lambda}$, where the $\hat{e}$ are the polarization vectors of the electric fields. The final results depend on $\theta$ the scattering angle. A general autocorrelation function will be denoted by $\Phi^{A}(q, \omega)=(A(\mathbf{q}, \omega) \mid A(\mathbf{q}))$.

\section{A. Macroscopic dielectric models}

In liquids and gases the spatially averaged but still thermodynamically fluctuating dielectric function is connected to for example the fluctuating density via a thermodynamic derivative ${ }^{4}$

$$
\left.\delta \epsilon_{i j}(\mathbf{r}, t)=\frac{\partial \epsilon}{\partial n}\right)_{T} \delta n(\mathbf{r}, t) \delta_{i j} .
$$

Temperature fluctuations, which could also enter in Eq. (2) via $\partial \epsilon / \partial T)_{n}$ are generally found to be negligible. ${ }^{2}$ Therefore temperature fluctuations will be neglected throughout this paper. These scalar fluctuations do not change the direction of polarization. No depolarized spectra, i.e., $I^{H V}$ or $I^{V H}$ or $I^{H H}(\theta=\pi / 2)$, are expected.

In a glass dielectric fluctuations are coupled to the strain via the elasto-optic or Pockels constants ${ }^{5}$

$$
\begin{aligned}
& \delta \epsilon_{i j}(\mathbf{r}, t)=a_{1} u_{i j}(\mathbf{r}, t)+a_{2} u_{l l}(\mathbf{r}, t) \delta_{i j}, \\
& u_{i j}=\frac{1}{2}\left(\nabla_{i} u_{j}+\nabla_{j} u_{i}\right),
\end{aligned}
$$

$u_{i j}(\mathbf{r}, t)$ is the strain tensor and $u_{l l}=-\delta n(\mathbf{r}, t) / n$ the rela- tive density variation. This ansatz, e.g., leads to depolarized light scattering from transverse phonons

$$
I^{H V}(q, \omega) \propto \frac{\left|a_{1}\right|^{2}}{4 n^{2}} \frac{q^{2}}{\omega^{2}} \cos ^{2} \frac{\theta}{2} S_{t}(q, \omega) .
$$

The limit $\omega \rightarrow 0$ poses no problem in Eq. (4), because in a solid the transverse current fluctuation function $S_{t}(q, \omega) \propto \omega^{2}$ also vanishes. In a liquid Eq. (3) has to be replaced by ${ }^{5}$

$$
\begin{aligned}
& \delta \epsilon_{i j}(\mathbf{r}, t)=\lambda_{1} v_{i j}(\mathbf{r}, t)+a_{2} \frac{-\delta n(\mathbf{r}, t)}{n} \delta_{i j}, \\
& v_{i j}=\frac{1}{2 n}\left(\nabla_{i} j_{j}+\nabla_{j} j_{i}\right),
\end{aligned}
$$

where $\mathbf{j}$ is the current density and the elasto-optic constant $\lambda_{1}$ replaces $a_{1}$. A comparison of both descriptions leads to $a_{1} \propto \lambda_{1} \omega$. Within the viscoelastic theory of a liquid a solid is obtained if frequencies larger than the inverse of the viscoelastic relaxation time $\tau$ are considered. ${ }^{18}$ In the context of the liquid to glass transition this relaxation process is called $\alpha$-relaxation. ${ }^{10}$ From the above two pictures it can be seen, that $\lambda_{1}$ and $a_{1}$ define two limits, $\omega \tau \ll 1$ and $\omega \tau \gg 1$, respectively, of a generalized frequency dependent Pockels constant.

\section{B. Microscopic dielectric model}

In systems of spherical particles with scalar molecular polarizability $\alpha_{0} \delta_{i j}$ depolarized spectra are caused by multiple scattering of photons by at least pairs of density fluctuations. ${ }^{8}$ In order to show that this mechanism leads to frequency dependent generalizations of the thermodynamic derivatives in Eq. (2), a simplified version of the dielectric model of Felderhof ${ }^{13}$ is used.

Assuming an infinite, homogeneous and isotropic system of point particles with scalar polarizability $\alpha_{0} \delta_{i j}$ Felderhof ${ }^{13}$ arrives at Eq. (1), where

$$
\begin{aligned}
\delta \epsilon_{i j}(\mathbf{r}, t)= & 4 \pi \alpha_{0}\left(\frac{\epsilon_{0}+2}{3}\right)^{2}\left\{\delta n(\mathbf{r}, t) \delta_{i j}\right. \\
& +\alpha_{0} \delta\left[\int d^{3} r^{\prime} \delta n(\mathbf{r}) U_{i j}^{k}\left(\mathbf{r}-\mathbf{r}^{\prime}\right) \delta n\left(\mathbf{r}^{\prime}\right)\right] \\
& \left.+o\left(\left[4 \pi \alpha_{0}\left(\frac{\epsilon_{0}+2}{3}\right)^{2} \delta n\right]^{3}\right)\right\}
\end{aligned}
$$

The formula breaks down at high enough frequencies for electronic effects to become important. $U_{i j}^{k}$ is an effective dipole radiation propagator for a medium with dielectric constant $\epsilon_{0}$

$$
U_{i j}^{k}(\mathbf{r})=\left(\frac{\epsilon_{0}+2}{3}\right)^{2} \frac{1}{\epsilon_{0}}\left[2\left(\frac{4 \pi}{3}\right)^{2} \alpha_{0} n \delta_{i j} \delta(\mathbf{r})+L_{i j}^{k_{L}}(\mathbf{r})\right] .
$$

Two further simplifying approximations shall be performed. The scalar part yields a renormalization of the first order term. It is smaller than this term by a factor of $\alpha_{0} n \ll 1$ and does not introduce any qualitatively new effects. Therefore it will be dropped. Furthermore, the dipole radiation propagator $L_{i j}^{k_{L}}(\mathbf{r})$ is often approximated by the static dipole induced dipole propagator ${ }^{19}$ 


$$
\frac{1}{\epsilon_{0}} L_{i j}^{k_{L}}(\mathbf{k}) \rightarrow 4 \pi F_{i j}^{\prime}(\mathbf{k})=-4 \pi\left(\frac{k_{i} k_{j}}{k^{2}}-\frac{1}{3} \delta_{i j}\right) .
$$

With these simplifications a spatial Fourier transformation of Eq. (6) leads to

$$
\begin{aligned}
\delta \epsilon_{i j}(\mathbf{q}, t)= & \alpha \delta n(\mathbf{q}, t) \delta_{i j}+\alpha^{2} \int \frac{d^{3} k}{(2 \pi)^{3}} \delta n(\mathbf{q}-\mathbf{k}, t) \\
& \times\left(\frac{1}{3} \delta_{i j}-\hat{k}_{i} \hat{k}_{j}\right) \delta n(\mathbf{k}, t)+O\left[(\alpha \delta n)^{3}\right] \\
& +O\left[\alpha_{0} n(\alpha \delta n)^{2}\right] .
\end{aligned}
$$

The coefficient $\alpha$ is defined by

$$
\alpha=\left(\frac{\partial \epsilon_{0}}{\partial n}\right)_{T}=4 \pi \alpha_{0}\left(\frac{\epsilon_{0}+2}{3}\right)^{2}
$$

where $\alpha$ depends on the dielectric model, which to this order reduces to the Clausius Mossotti equation for the dielectric function ${ }^{13}$

$$
\frac{\epsilon_{0}-1}{\epsilon_{0}+2}=\frac{4 \pi}{3} \alpha_{0} n
$$

The aim of describing light scattering at the liquid to glass transition requires the dielectric model to be applicable to dense systems. ${ }^{20}$ In this paper the qualitative features of light scattering in supercooled liquids shall be explored. A more realistic dielectric model will only have different effective polarizability prefactors as long as an expansion of $\delta \epsilon_{i j}$ in products of density fluctuations is possible.

\section{DERIVATION OF ELASTO-OPTIC CONSTANTS AND SCATTERING SPECTRA}

A projection operator formalism ${ }^{17}$ calculates the hydrodynamic poles contained in the dielectric spectra. The formalism is summarized in Appendix A. In order to simplify the interpretation of the spectra caused by the fluctuations of Eq. (7) generalized elasto-optic constants shall be introduced first.

Specifying $\delta X$ in formula (A1) to $\delta \epsilon_{i j}$ leads to

$$
\begin{aligned}
\left\langle\delta \epsilon_{i j}^{*}(\mathbf{q}, z)\right\rangle= & A_{i j}(\mathbf{q})\left\langle\delta n^{*}(\mathbf{q}, z)\right\rangle \\
& +B_{\alpha i j}(\mathbf{q}, z)\left\langle j_{\alpha}^{*}(\mathbf{q}, z)\right\rangle, \\
A_{i j}(\mathbf{q})=\alpha \delta_{i j} & +\frac{\alpha^{2}}{(n(q) \mid n(q))} \int \frac{d^{3} k}{(2 \pi)^{3}} F_{i j}^{\prime}(\mathbf{k}) \\
\times & (n(\mathbf{q}-\mathbf{k}) n(\mathbf{k}) \mid n(\mathbf{q})), \\
B_{\alpha i j}(\mathbf{q}, z)=- & \alpha^{2} \int \frac{d^{3} k}{(2 \pi)^{3}} F_{i j}^{\prime}(\mathbf{k}) \\
\times & \frac{\left(n(\mathbf{q}-\mathbf{k}) n(\mathbf{k})\left|R^{\prime}(z) L\right| j_{\alpha}(\mathbf{q})\right)}{\left(j_{\alpha}(q) \mid j_{\alpha}(q)\right)} .
\end{aligned}
$$

Only the leading term in $q$ will be kept because of $q a \leqslant 1$. Because of the isotropy of the considered system the tensor structure of $B_{\alpha i j}(\mathbf{q}, z)$ can be given explicitly, if one remembers, that $F_{i j}^{\prime}$ is symmetric and traceless,

$$
\begin{gathered}
B_{\alpha i j}(\mathbf{q}, z)=\alpha^{2}(n / 2)\left(\frac{2}{3} q_{\alpha} \delta_{i j}-q_{i} \delta_{\alpha j}-q_{j} \delta_{\alpha i}\right) T(z), \\
T(z)=\lim _{q \rightarrow 0} \frac{3 / 2}{n q_{3}} \int \frac{d^{3} k}{(2 \pi)^{3}}\left(\frac{1}{3}-\hat{k}_{3}^{2}\right) \\
\times \frac{\left(n(\mathbf{q}-\mathbf{k}) n(\mathbf{q})\left|R^{\prime}(z) L\right| j_{3}(\mathbf{q})\right)}{\left(j_{3}(q) \mid j_{3}(q)\right)}
\end{gathered}
$$

Recognizing the velocity gradient tensor and applying the particle conservation law

$$
\begin{aligned}
q_{\alpha}\left\langle J_{\alpha}^{*}(\mathbf{q}, z)\right\rangle=\left\langle L \delta n^{*}(\mathbf{q}, z)\right\rangle= & z\left\langle\delta n^{*}(\mathbf{q}, z)\right\rangle \\
& +\left\langle\delta n^{*}(\mathbf{q}, t=0)\right\rangle
\end{aligned}
$$

the generalization of (5) can be obtained:

$$
\begin{aligned}
\left\langle\delta \epsilon_{i j}^{*}(\mathbf{q}, z)\right\rangle= & a_{2}(z)\left[-\frac{\left\langle\delta n^{*}(\mathbf{q}, z)\right\rangle}{n}\right] \delta_{i j} \\
& +\lambda_{1}(z)\left\langle v_{i j}^{*}(\mathbf{q}, z)\right\rangle \\
& +\frac{\alpha^{2} n}{3} T(z)\left\langle\delta n^{*}(q, t=0)\right\rangle \delta_{i j},
\end{aligned}
$$

where the frequency dependent coupling coefficients are

$$
\begin{aligned}
& a_{2}(z)=-\alpha n\left[1+\frac{\alpha n}{3} z T(z)\right], \\
& \lambda_{1}(z)=i(\alpha n)^{2} T(z) .
\end{aligned}
$$

Introducing a frequency dependent coupling $T(z)$ between $\delta \epsilon_{i j}$ and $\delta n$ or $\delta v_{i j}$ also leads to the direct observation of the nonhydrodynamic dynamics of $T(z)$ in $\delta \epsilon_{i j}$.

Neglecting vacancy diffusion processes the connection between the strain and the velocity gradient tensor runs ${ }^{21}$

$$
v_{i j}(\mathbf{r}, t)=\dot{u}_{i j}(\mathbf{r}, t) \text {. }
$$

This leads to the generalization of Eq. (3) as

$$
\begin{aligned}
\left\langle\delta \epsilon_{i j}^{*}(\mathbf{q}, z)\right\rangle= & a_{2}(z)\left[-\frac{\left\langle\delta n^{*}(\mathbf{q}, z)\right\rangle}{n}\right] \delta_{i j} \\
& +a_{1}(z)\left\langle u_{i j}^{*}(\mathbf{q}, z)\right\rangle \\
& +\frac{\alpha^{2} n}{3} T(z)\left\langle\delta n^{*}(q, t=0)\right\rangle \delta_{i j} \\
& +(\alpha n)^{2} T(z)\left\langle u_{i j}^{*}(\mathbf{q}, t=0)\right\rangle,
\end{aligned}
$$

where $T(z)$ and $a_{2}(z)$ have been defined above and $a_{1}(z)$ equals

$$
a_{1}(z)=(\alpha n)^{2} z T(z)
$$

It is interesting to note, that the $t=0$ limit of the one relevant coupling function $T(t)$ can be calculated exactly. In a system where the interparticle potential is sufficiently smooth, so that $k^{3}\left(S_{k}-1\right) \rightarrow 0$ for $k \rightarrow \infty \quad\left(S_{k}\right.$ the static structure factor), the simple result $T(t=0)=\frac{2}{3}$ is obtained (see Appendix B).

From the formula (7) for the dielectric fluctuations the light scattering intensity $I^{2 \lambda^{\prime}}$ can be obtained 
$\Phi^{\circ}(q, t)$

$$
\begin{aligned}
= & \left(\epsilon_{i j}(\mathbf{q})|R(t)| \epsilon_{k l}(\mathbf{q})\right) \\
= & \alpha^{2}(n(\mathbf{q})|R(t)| n(\mathbf{q})) \delta_{i j} \delta_{k l} \\
& +2 \alpha^{3} \delta_{i j} \int \frac{d^{3} k}{(2 \pi)^{3}} F_{k l}^{\prime}(\mathbf{k})(n(\mathbf{q})|R(t)| n(\mathbf{q}-\mathbf{k}) n(\mathbf{k})) \\
& +\alpha^{4} \int \frac{d^{3} k}{(2 \pi)^{3}} \frac{d^{3} k^{\prime}}{(2 \pi)^{3}} F_{i j}^{\prime}(\mathbf{k}) F_{k l}^{\prime}\left(\mathbf{k}^{\prime}\right) \\
& \times\left(n(\mathbf{q}-\mathbf{k}) n(\mathbf{k})|R(t)| n\left(\mathbf{q}-\mathbf{k}^{\prime}\right) n\left(\mathbf{k}^{\prime}\right)\right) .
\end{aligned}
$$

Using similar symmetry considerations as when studying $B_{\alpha i j}$ it is straightforward to obtain the total scattered intensities $\bar{T}^{\lambda \lambda^{\prime}}=S(d \omega / 2 \pi)\left(\epsilon_{\lambda \lambda^{\prime}}|R(\omega)| \epsilon_{\lambda \lambda^{\prime}}\right)$ from Eq. (14)

$$
\begin{aligned}
\bar{I}^{V V}(q, \theta)= & \alpha^{2} \frac{N}{k T} S_{q}+\frac{4}{3} \alpha^{4} \Phi^{F^{\prime} n^{2}}(t=0), \\
\bar{I}^{H V}(q, \theta)= & \bar{I}^{V H}(q, \theta)=\alpha^{4} \Phi^{F^{\prime} n^{2}}(t=0), \\
\bar{I}^{H H}(q, \theta)= & \alpha^{2} \cos ^{2} \theta \frac{N}{k T} S_{q} \\
& +\alpha^{4}\left(1+\frac{1}{3} \cos ^{2} \theta\right) \Phi^{F^{\prime} n^{2}}(t=0),
\end{aligned}
$$

where, because of $R(t=0)=1$, the static structure factor $S_{q}=(k T / N) \Phi^{n}(q, t=0)$ appears. Because of $q a \ll 1$ it can be replaced by its thermodynamic limit $\left.S_{q \rightarrow 0}=k T \partial n / \partial p\right)_{T}$. The anisotropic spectrum $\Phi^{F^{\prime} n^{2}}$ has a nonzero value for $q \rightarrow 0 .{ }^{16}$

$$
\begin{aligned}
\Phi^{F^{\prime} n^{2}}(t=0)= & \lim _{q \rightarrow 0} \frac{3}{4} \int \frac{d^{3} k}{(2 \pi)^{3}} \frac{d^{3} k^{\prime}}{(2 \pi)^{3}}\left(\frac{1}{3}-\hat{k}_{3}^{2}\right)\left(\frac{1}{3}-\hat{k}_{3}^{\prime 2}\right) \\
& \times\left(n(\mathbf{q}-\mathbf{k}) n(\mathbf{k}) \mid n\left(\mathbf{q}-\mathbf{k}^{\prime}\right) n\left(\mathbf{k}^{\prime}\right)\right) .
\end{aligned}
$$

Equations (14) and (15) were used before in liquids and gases; the hydrodynamic pole contributions at the glass transition however have not been treated correctly up to now. Using the projector formalism (A2) and applying similar transformations as when deriving the elasto-optic constants a simple but lengthy calculation leads to the spectra:

$$
\begin{aligned}
\bar{I}^{\nu^{\prime}{ }^{\prime}(q, z, \theta)=} & {\left[-\frac{a_{2}(z)}{n}\right]^{2} \Phi^{n}(q, z)+\frac{4}{3} \alpha^{4} \Phi^{F^{\prime} n^{2}}(z) } \\
& +\left[\frac{2}{3} \alpha^{3} n T(z)+\left(\frac{\alpha^{2} n}{3}\right)^{2} z T^{2}(z)\right] \\
& \times \Phi^{n}(q, t=0), \\
\bar{I}^{H V}(q, z, \theta)= & \bar{I}^{V H}(q, z, \theta) \\
= & \alpha^{4} \Phi^{F^{\prime} n^{2}}(z)-\frac{q^{2}}{4} \cos ^{2} \frac{\theta}{2}\left[\frac{\lambda_{1}(z)}{n}\right]^{2} \Phi^{t}(q, z) \\
= & \alpha^{4} \Phi^{F^{\prime} n^{2}}(z)+\frac{q^{2}}{4} \cos ^{2} \frac{\theta}{2}\left[\frac{a_{1}(z)}{z n}\right]^{2} \Phi^{2}(q, z), \\
\bar{I}^{H H}(q, z, \theta)= & \left\{\frac{\left[a_{2}(z)+\frac{1}{2} a_{1}(z)\right] \cos \theta+\frac{1}{2} a_{1}(z)}{n}\right\}^{2} \\
& \times \Phi^{n}(q, z)+\alpha^{4}\left(1+\frac{1}{3} \cos { }^{2} \theta\right) \Phi^{F^{\prime} n^{2}}(z) \\
& +\left[\frac{1}{4} \alpha^{4} n^{2} z T^{2}(z)\left(1+\frac{1}{3} \cos \theta\right)^{2}\right. \\
& \left.-\alpha^{3} n T(z) \cos \theta\left(1+\frac{1}{3} \cos \theta\right)\right] \Phi^{n}(q, t=0),
\end{aligned}
$$

$$
\begin{aligned}
\Phi^{F^{\prime} n^{2}}(z)= & \lim _{q \rightarrow 0} \frac{3}{4} \int \frac{d^{3} k}{(2 \pi)^{3}} \frac{d^{3} k^{\prime}}{(2 \pi)^{3}}\left(\frac{1}{3}-\hat{k}_{3}^{2}\right)\left(\frac{1}{3}-\hat{k}_{3}^{\prime 2}\right) \\
& \times\left(n(\mathbf{q}-\mathbf{k}) n(\mathbf{k})\left|R^{\prime}(z)\right| n\left(\mathbf{q}-\mathbf{k}^{\prime}\right) n\left(\mathbf{k}^{\prime}\right)\right),
\end{aligned}
$$

where $a_{1}(z), a_{2}(z)$, and $\lambda_{1}(z)$ are the already defined frequency dependent elasto-optic constants. Directly starting from an ansatz like Eq. (3) with frequency dependent couplings misses the terms containing the dynamics of the coupling functions alone. The total intensities in Eq. (15) can again be obtained from Eq. (17). It is worth noting, that the coupling to the transversal current does not contribute to the total scattered intensity. $\bar{I}^{H V}(q, \theta)$ is independent of the scattering angle, whereas $\bar{I}^{H V}(q, z, \theta)$ varies with $\theta$.

The hydrodynamic density and transverse current fluctuation functions in viscous liquids enter the formula (17). The standard results of viscoelastic theory ${ }^{22}$ shall be summarized for completeness:

$$
\begin{gathered}
\Phi^{n}(q, z)=-\Phi^{n}(q, t=0)\left(z+\frac{q^{2}}{m n} K_{l}(z)\right) / \\
{\left[z^{2}+z \frac{q^{2}}{m n} K_{l}(z)-q^{2} c_{0}^{2}\right],}
\end{gathered}
$$

where the longitudinal memory kernel, a generalized viscosity, is given by the longitudinal components of the stress tensor $\tau_{i j}$ :

$$
K_{l}(z)=\frac{1}{V} \lim _{q \rightarrow 0}\left(\tau_{l l}(\mathbf{q})\left|R^{\prime}(z)\right| \tau_{l l}\right) .
$$

Again temperature fluctuations are neglected: the diffusive heat pole $\propto 1 /\left(z+i q^{2} D_{T}\right)$, where $D_{T}=\kappa / m n c_{p}$ is determined by the heat conductance $\kappa$, is dropped in Eq. (19). It has only to be added to the spectra discussed below. In the transverse current correlator another memory function shows up:

$$
\begin{aligned}
& \Phi^{t}(q, z)=-\frac{N}{m}\left[z+\frac{q^{2}}{m n} K_{s}(z)\right], \\
& K_{s}(z)=\frac{1}{V} \lim _{q \rightarrow 0}\left(\tau_{l t}(\mathbf{q})\left|R^{\prime}(z)\right| \tau_{l t}(\mathbf{q})\right) .
\end{aligned}
$$

Except for assuming the validity of formulas (1) and (7) no further approximations are necessary for deriving the results of this chapter.

\section{DISCUSSION FOR A SUPERCOOLED LIQUID}

This qualitative discussion shall point out the general, still exact contents of the derived microscopic formula for the Pockels constants and the spectra.

The memory functions and generalized coupling functions of the preceding chapter have the reduced dynamics $R^{\prime}(z)$, which contains no hydrodynamic poles. It still varies on a diverging relaxation time scale $\tau$, which becomes large compared to any experimental time scale when supercooling the liquid below the glass transformation temperature $T_{g}{ }^{10}$ In the following discussion mainly the dynamics on this strongly varying time scale $\tau$ shall be considered. The change of $\tau$ with temperature causes the qualitative change of the light scattering spectra from liquid to solid-like. In a liquid $\tau$ 
is of the order of the microscopic time $\tau \approx t_{0} \approx 10^{-12} \mathrm{~s}$, whereas in a glass $\tau$ exceeds seconds. Any faster, e.g., microscopic, relaxation time shall be denoted by $t_{0}$. Whenever $\omega t_{0} \approx 1$ is not included explicitly, the frequency window is restricted by $\omega t_{0} \ll 1$.

As experimental time scale of Brillouin scattering $\omega \approx q c$ is taken, where $c$ is the velocity of sound and is considered to be little dependent on temperature and frequency. In the liquid region, given by $q c \tau \ll 1$, the zero frequency limit can be taken in all relaxation functions, which do not contain hydrodynamic poles. The known thermodynamic derivatives and transport coefficients are obtained. In the low temperature or glass region, given by $q c \tau \gg 1$, the $\alpha$ proces is taken to be not resolved because of finite experimental resolution power. In the glass the $\alpha$ relaxation is taken to be frozen out and is given by an elastic line. Thermodynamic derivatives like the specific heat $c_{p}(T)$ will show a step when going from the high to the low temperature side, because of the moving of the $\alpha$ peak through the experimental frequency window. ${ }^{10}$

Keeping $\omega t_{0} \ll 1$ in mind the mentioned limits in $K_{l}(z)$ and $K_{s}(z)$ are found to be: ${ }^{22}$

$$
\begin{aligned}
& K_{l}(z) \rightarrow i \eta_{l} \text { for } z \tau \ll 1 \\
& K_{l}(z) \rightarrow \frac{-1}{z}\left(M_{\infty}-M_{0}\right) \quad \text { for } z \tau \gg 1, \\
& K_{s}(z) \rightarrow i \eta_{s} \text { for } z \tau \ll 1 \\
& K_{s}(z) \rightarrow \frac{-1}{z} G_{\infty} \quad \text { for } z \tau \gg 1 .
\end{aligned}
$$

The longitudinal and shear viscosities $\eta_{l}$ and $\eta_{s}$ are connected to the high frequency longitudinal and shear modulus respectively via averaged relaxation times $\tau_{l}$ and $\tau_{s}{ }^{22}$

$$
\eta_{l}=\tau_{l}\left(\boldsymbol{M}_{\infty}-\boldsymbol{M}_{0}\right) \quad \eta_{s}=\tau_{s} G_{\infty},
$$

where, for example, $\tau_{s}=\tau \int_{0}^{\infty} d(t / \tau) K_{s}(t / \tau) / K_{s}(t / \tau \rightarrow 0)$ differs from $\tau$ by some factor of the order of 1 .

Denoting in analogy the high frequency limit of the $\alpha$ relaxation in $T(z)$ as $T_{\infty}$ and the corresponding relaxation time as $\tau_{T}$ the following limits for $T(z)$ are obtained:

$$
\begin{aligned}
& T(z) \rightarrow i \tau_{T} T_{\infty} \quad \text { for } z \tau \ll 1, \\
& T(z) \rightarrow \frac{-1}{z} T_{\infty} \quad \text { for } z \tau \gg 1 .
\end{aligned}
$$

Because $T(z)$ is no autocorrelation function, it can only be said, that $T^{\prime \prime}(\omega) / \omega$ is an even, real function of $\omega$; the sign of $T_{\infty}$ however is not fixed. The experimentally observed strong variation of $\tau_{t}$ and $\tau_{s}$ with temperature also applies to $\tau_{T}$, whereas $T_{\infty}$ is expected to vary only little with temperature, as found for $G_{\infty}$ and $M_{\infty} .^{3}$

In the same manner the area of the $\alpha$ relaxation peak in the anisotropic spectrum $\Phi^{F^{\prime} n^{2}}$ is denoted by $\Phi_{\infty}^{F^{\prime} n^{2}}$ and the corresponding relaxation time as $\tau_{\text {aniso }}$.

In the following discussion a small damping of the phonons in the glass shall be taken into account by adding as next to leading order contribution $i m n \Gamma_{l}$ and $i m n \Gamma_{s}$ to the limits of $K_{l}(z)$ and $K_{s}(z)$, respectively, for $z \tau \gg 1$.

\section{A. Elasto-optic constants}

In the limit $\omega \tau \ll 1, a_{2}$ reduces to the expected thermodynamic derivative ${ }^{4}$

$$
a_{2}(z) \rightarrow-n \alpha=-n\left(\frac{\partial \epsilon}{\partial n}\right)_{T} \quad \text { for } z \tau \ll 1 .
$$

In the glass $\omega \tau \gg 1$ however the frozen structure contributes to the scalar scattering:

$$
a_{2}(z) \rightarrow-n \alpha\left(1-\frac{\alpha n}{3} T_{\infty}\right) \text { for } z \tau \gg 1 .
$$

The transverse current couples only weakly, $\tau_{T} \approx \tau_{s} \propto \eta_{s}$, to dielectric fluctuations in the liquid, whereas in the glass a finite coupling is obtained ${ }^{5}$

$$
\begin{aligned}
& \lambda_{1}(z) \rightarrow-(\alpha n)^{2} T_{\infty} \tau_{T} \text { for } z \tau \ll 1, \\
& a_{1}(z) \rightarrow-(\alpha n)^{2} T_{\infty} \text { for } z \tau \gg 1 .
\end{aligned}
$$

Comparing $a_{1} / \omega$ with $\lambda_{1}$ one sees that in the liquid the coupling coefficient of the transverse current is reduced by a factor $\omega \tau_{T} \ll 1 . \lambda_{1}$ vanishes with increasing temperature proportionally to the viscosity. For known $\partial \epsilon / \partial n)_{T}$ Eqs. (27) and (29) allow to measure $T_{\infty}$ and to check the underlying dielectric model.

\section{B. Total scattered intensities}

Summing up all spectral contributions without the restriction $\omega t_{0} \ll 1$ the total scattered intensities [Eq. (15)] are obtained. In order $\alpha^{2}$ the light scattering is scalar, whereas the $\alpha^{4}$ contribution is recognized to be an anisotropic scattering process ${ }^{1}$

$$
\bar{I}^{\text {aniso }} \bar{I}^{\text {iso }}=\alpha^{2} \frac{\Phi^{F^{\prime} n^{2}}(t=0)}{(N / k T) S_{q}} .
$$

Experimental ratios of $\bar{I}^{\text {aniso }} \bar{I}^{\text {iso }}$ in systems of optically isotropic particles lie between 0.1 (Ref. 23) and $10^{-3},{ }^{24}$ indicating that the expansion in $(\alpha \delta n)$ is possible. Only the first cited value corresponds to a system at the liquid to glass transition.

\section{Spectral shapes}

Regardless of the size of $\omega \tau$ the spectra in the regime $\omega \gg c q$ simplify. The restriction $\omega t_{0} \ll 1$ is put aside for discussing this low frequency translational Raman region.

For $\omega \gg c q$ the density and the transverse current correlators for hydrodynamic fluctuations have narrowed to elastic lines, which lie outside of the specified frequency window. Then only the anisotropic scattering contributes leading to a frequency independent depolarization ratio ${ }^{23}$

$$
I^{H v}(\omega, \theta) / I^{v v}(\omega, \theta)=\frac{3}{4} \text { for } \omega \gg c q .
$$

The spectra in the frequency range $\omega<\approx c q$, especially $I^{H V}$, change drastically from liquid to solid like when supercooling the liquid. In the following $I^{V V}$ and $I^{H V}$ are discussed, because $I^{H H}$ can be obtained from them. The series of Figs. $1-4$ is meant to clarify the discussion. Polarized $I^{V V}$ and depolarized $I^{H V}$ spectra are shown for three different values of $\tau$. Other parameters like relative intensities, intensity units etc., are fixed in Figs. $1-4$. Frequencies are given in 
units of $1 / t_{0}$, a typical frequency of translational Raman bands. The typical Brillouin frequency, i.e., the thermodynamic sound pole position was chosen to lie at $c_{0} q t_{0}=0.001$. A list of further parameters can be found in Sec. V B. The diffusive heat pole is not included in the pictures.

In the liquid the polarized spectrum is dominated by the hydrodynamic structure factor $S_{q}(\omega)$ $=2(N / k T) \Phi^{n^{*}}(q, \omega)$ which sits on a very weak broad (depolarized) background from the anisotropic scattering. The frequency dependent coupling $T(\omega)$ $=2 T^{\prime \prime}(\omega)=2 \operatorname{Im}[T(z=\omega+i 0)]$ contributes another very weak constant background for $\omega \tau \ll 1$,

$$
\begin{aligned}
\bar{I}^{\prime r}(\omega, q)= & \alpha^{2} \frac{N}{k T} S_{q}(\omega)+\frac{4}{3} \alpha^{4} \Phi^{F^{\prime} n^{2}}(\omega) \\
& +\frac{2}{3} \alpha^{3} n T_{\infty} \tau_{T} \frac{N}{k T} S_{q} \quad \text { for } \omega \approx c q \ll 1 / \tau, \\
\bar{I}^{v v}(\omega, q)= & \alpha^{2} \frac{N}{k T} S_{q} \delta(\omega)+\frac{4}{3} \alpha^{4} \Phi^{F^{\prime} n^{2}}(\omega) \\
& \text { for } c q \ll \omega \ll 1 / \tau .
\end{aligned}
$$

For the dominant term in Eqs. (32) the ansatz $\delta \epsilon=(\partial \epsilon / \partial n) \delta n$ is obtained. Figure 1(a) shows a typical hydrodynamic spectrum for $c_{0} q \tau \approx 0.016$. In Fig. 2(a) for $c_{0} q \tau \approx 0.2$ viscoelastic effects can be seen in the stronger damping and the increased central intensity.

The depolarized spectrum $I^{H V}$ in a liquid consists mainly of the broad background from anisotropic scattering which varies on scale $1 / \tau_{\text {aniso }}$. A further negative central line, which is generally called Rytov Dip, ${ }^{14}$ is also found.

$$
\begin{aligned}
\bar{I}^{H V}(\omega, q)= & \alpha^{4} \Phi^{F^{\prime} n^{2}}(\omega)-\left(\frac{q n}{2} \cos \frac{\theta}{2}\right)^{2} \alpha^{4} T_{\infty}^{2} \tau_{T}^{2} \\
& \times \frac{1}{k T} S_{t}(q, \omega) \quad \text { for } \omega \tau \ll 1,
\end{aligned}
$$

i.e., a line given by the hydrodynamic transverse current fluctuation function

$$
S_{t}(q, \omega)=\frac{2 k T N}{m} \frac{\left(\eta_{s} / m n\right) q^{2}}{\omega^{2}+\left[\left(\eta_{s} / m n\right) q^{2}\right]^{2}}
$$

is cut out of the anisotropic line. The total intensity of this line is

$$
I^{\mathrm{dip}}=\left(\frac{1}{2} \cos \frac{\theta}{2}\right)^{2}\left(q c_{0} \tau_{r}\right)^{2}\left(T_{\infty} \alpha n\right)^{2} I^{\text {iso }}
$$

and its width varies with $\left(\eta_{s} / m n\right) q^{2}$. Whether this negative line can be observed experimentally depends crucially on the size of $\tau_{T}$; compare Figs. $1(\mathrm{~b})$ and $2(\mathrm{~b})$, where the upper halves of the anisotropic lines are shown. In Fig. 2(b), where $c_{0} q \tau \approx 0.2$, it can be observed on the frequency scale also used for studying the polarized spectrum. When $\tau$ is too small, as in Fig. $1(\mathrm{~b})$, where $c_{0} q \tau \approx 0.016$, an observation is difficult. There its width is smaller than $2 \times 10^{-5} / t_{0}$ and its intensity is roughly a factor of 100 smaller than in Fig. 2(b). The intensity which $\lambda_{1}^{2}(\omega) S_{t}(q, \omega)$ subtracts at $\omega t \ll 1$ is again added around $\omega \tau \approx 1$. Therefore at $\tau\left(\eta_{s} / m n\right) q^{2} \approx 1$ the simple interpretation on Eq. (33) as a dip does not apply anymore.
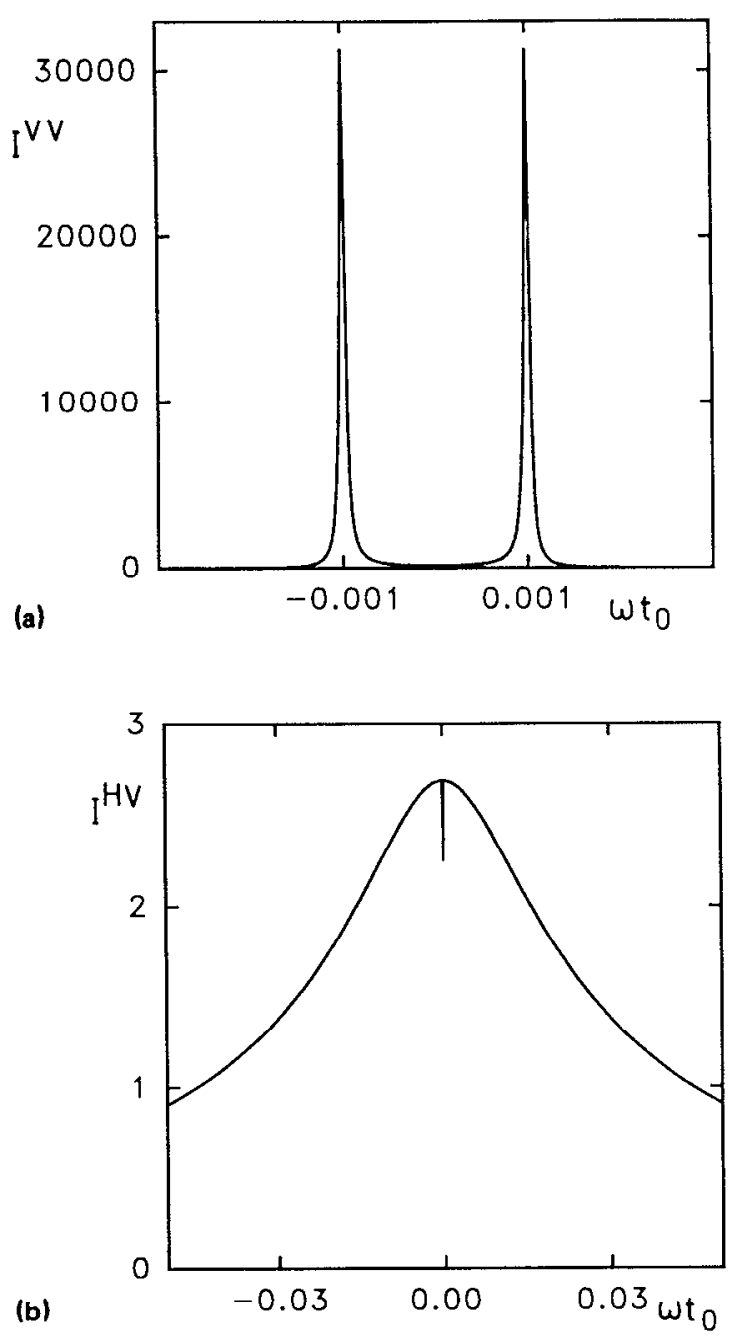

FIG. 1. Polarized $I^{V V}$ (a) and depolarized $I^{H V}$ (b) spectra of a liquid in the hydrodynamic regime. The $\alpha$ relaxation $\tau$ is $c_{0} q \tau \approx 0.016$. The thermal Rayleigh peak is not included.

In the glass at $\omega \tau \gg 1, S_{q}(\omega)$ shows another peak besides the phonon peaks and the (neglected) heat peak. This Mountain peak ${ }^{25}$ stems from the $\alpha$ peak of $K_{l}(z)$. Within the approximation (23) and for small $\Gamma_{l}$ it is given by an elastic line with weight $\left(M_{\infty}-M_{0}\right) / M_{0}$. Qualitatively $S_{q}(\omega)$ is given by

$$
\begin{aligned}
S_{q}(\omega)= & 2 S_{q}\left[\left(1-\frac{c_{0}^{2}}{c_{\infty}^{2}}\right) \pi \delta(\omega)\right. \\
& \left.+\frac{c_{0}^{2}}{c_{\infty}^{2}} \frac{\omega^{2} q^{2} \Gamma_{l}}{\left(\omega^{2}-c_{\infty}^{2} q^{2}\right)^{2}+\left(\omega q^{2} \Gamma_{l}\right)^{2}}\right] \quad \text { for } \omega \tau \gg 1 .
\end{aligned}
$$

Here only the general three peak structure of $S_{q}(\omega)$ for $\omega \tau>1$ is presented. $S_{t}(q, \omega)$ has a similar phonon contribution at the transverse sound velocity $c_{t}^{2}=G_{\infty} / m n$ :

$$
S_{t}(q, \omega)=2 \frac{N k T}{m}\left[\frac{\omega^{2} q^{2} \Gamma_{s}}{\left(\omega^{2}-c_{t}^{2} q^{2}\right)^{2}+\left(\omega q^{2} \Gamma_{s}\right)^{2}}\right]
$$

for $\omega \tau \gg 1$.

The phonon pole, i.e., the contents of the curly brackets shall be abbreviated by $S_{\mathrm{ph}}\left(c_{t}, \omega\right)$ in the following; similarly for 

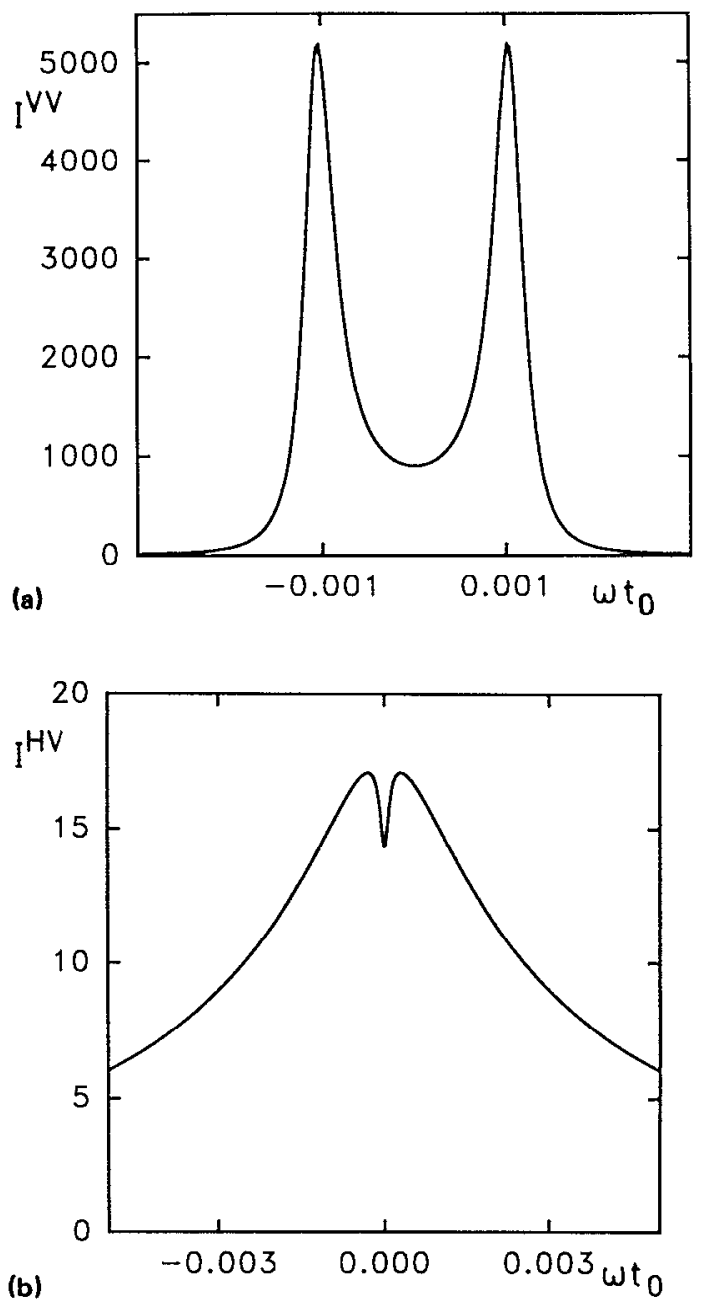

FIG. 2. Polarized $I^{V V}$ (a) and depolarized $I^{H V}$ (b) spectra of a liquid where viscoelastic effects are important. The increased $\alpha$ relaxation time $\tau$ is $c_{0} q \tau \approx 0.2$. The thermal Rayleigh peak is not included.

the longitudinal case. The spectra show further the anisotropic line, which has also narrowed drastically. The polarized spectrum of a glass is given by

$$
\begin{aligned}
\bar{I}^{V V}(\omega, q)= & \frac{4}{3} \alpha^{4} \Phi^{F^{\prime} n^{2}}(\omega)+\alpha^{2}\left[1-\frac{c_{0}^{2}}{c_{\infty}^{2}}\left(1-\frac{\alpha n}{3} T_{\infty}\right)^{2}\right] \\
& \times 2 \frac{N}{k T} S_{q} \pi \delta(\omega)+\alpha^{2}\left(1-\frac{\alpha n}{3} T_{\infty}\right)^{2} 2 \frac{N}{k T} \\
& \times S_{q} \frac{c_{0}^{2}}{c_{\infty}^{2}} S_{\mathrm{ph}}\left(c_{\infty}, \omega\right) \quad \text { for } \omega \tau \gg 1
\end{aligned}
$$

Figure $3(a)$ shows a polarized spectrum for $c_{0} q \tau \approx 126$. The corresponding depolarized spectrum of Fig. 3(b) is described by

$$
\begin{aligned}
\bar{I}^{H V}(\omega, q)= & \alpha^{4} \Phi^{F^{\prime} n^{2}}(\omega)+\frac{q^{2}}{4} \cos ^{2} \frac{\theta}{2}\left(\frac{\alpha^{2} n T_{\infty}}{\omega}\right)^{2} \\
& \times 2 \frac{N}{m} S_{\mathrm{ph}}\left(c_{t}, \omega\right) \quad \text { for } \omega \tau \gg 1
\end{aligned}
$$
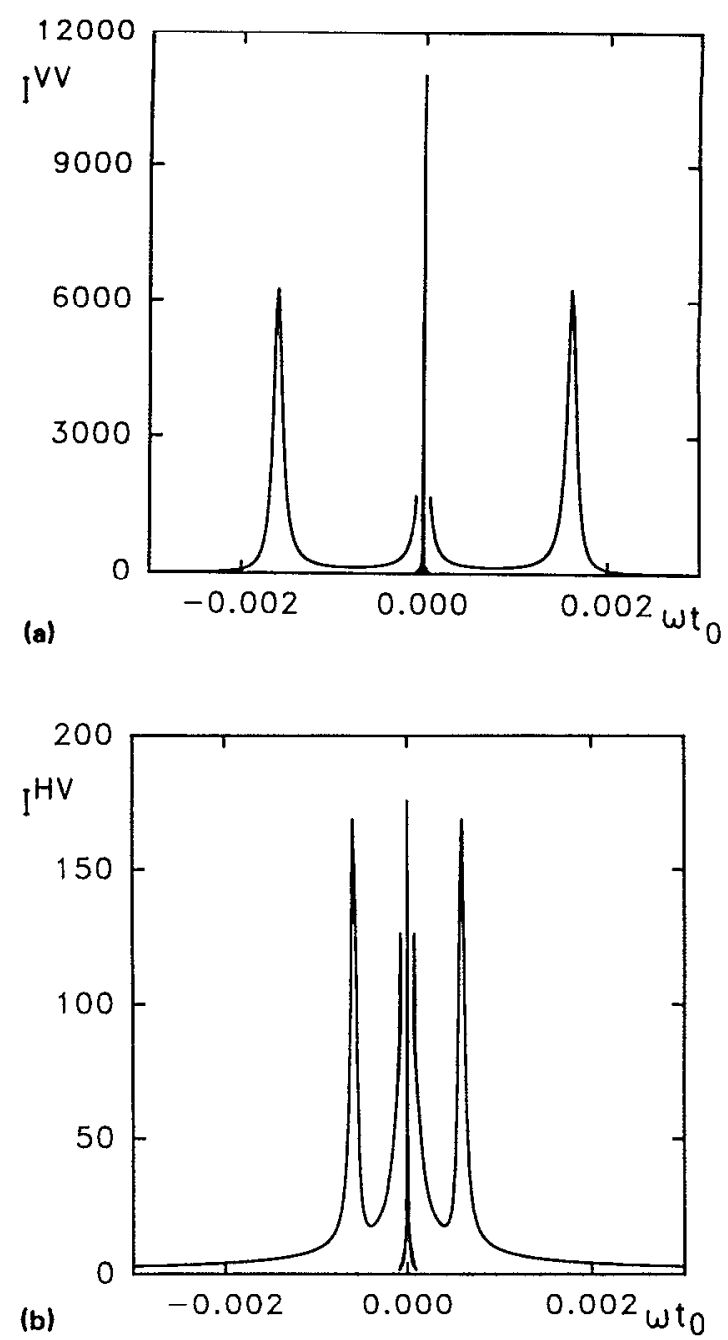

FIG. 3. Polarized $I^{V V}$ (a) and depolarized $I^{H V}$ (b) spectra of a glass. The $\alpha$ relaxation is effectively frozen out, $c_{0} q \tau \approx 126$. The polarized spectrum is dominated by the central Mountain peak, which has been rescaled by a factor of 50 in (a). The depolarized spectrum shows transverse phonons and the anisotropic spectrum, which has also been rescaled by a factor of 50 .

Remembering Eqs. (27) and (29) the elasto-optic constants $a_{1}$ and $a_{2}$ can be recognized. Neglecting the anisotropic line the phenomenological picture of coupling to the strain tensor is found. With formula (35) it is shown that the model for Brillouin scattering by density pairs, which has up to now only been applied to gases and simple low viscosity liquids, also leads to glass like spectra.

The anisotropic line has contributions on scale $1 / \tau$ but also shows the Rayleigh shoulder extending out to microscopic frequencies. Figure 4 shows a double logarithmic plot of the spectrum of Fig. 3(b). The frequency window ranges from $\omega \tau \approx 1$ to $\omega t_{0}>1$.

Within the specified dielectric model it is possible to connect various spectral intensities, if for example $\alpha=(\partial \epsilon / \partial n)_{T}$ has been measured independently.

The Landau-Placzek ratio of the central to both phonon peaks in the isotropic spectrum can be obtained from Eq. (34) in the glass 


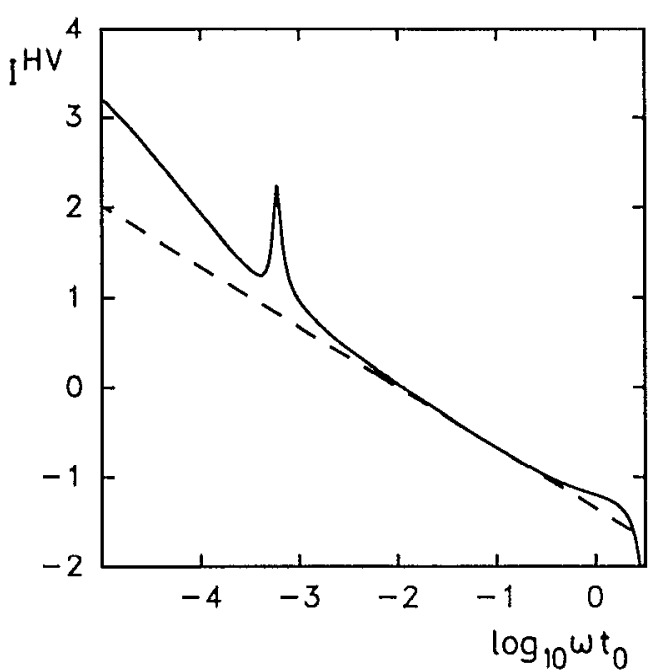

FIG.4. Double logarithmic plot of the depolarized spectrum of Fig. 3(b) showing the anisotropic spectrum and the transverse phonon peaks. The $\alpha$ relaxation time lies at $\tau \approx 10^{51} t_{0}$, the $\beta$ relaxation scale $t_{o}$ equals $t_{g} \approx 10^{32} t_{0}$. The dashed line corresponds to the asymptotic behavior $\Phi^{\prime \prime}(\omega) \propto \omega^{0073}$.

$$
I_{c}^{\text {iso }} / I_{\mathrm{ph}}^{\text {iso }}=\left(\frac{c_{\infty} / c_{0}}{1-(\alpha n / 3) T_{\infty}}\right)^{2}-1 \quad \text { for } \omega \tau \gg 1 .
$$

The neglect of the heat diffusion pole in $S_{q}(\omega)$ does not lead to the correct result $\bar{I}_{c}^{\text {iso }} \bar{I}_{\mathrm{ph}}^{\text {iso }}=c_{v} / c_{p}-1$ in the liquid. In a glass this correction to Eq. (36) can be neglected. ${ }^{2}$

The relative intensity of the longitudinal and transverse phonon peaks is

$I_{\mathrm{ph}}^{H V} / I_{\mathrm{ph}}^{\prime \nu}=\frac{1}{4} \cos ^{2} \frac{\theta}{2}\left(\frac{c_{\infty}}{c_{t}}\right)^{2}\left(\frac{\alpha n T_{\infty}}{1-(\alpha n / 3) T_{\infty}}\right)^{2}$ for $\omega \tau \gg 1$.

The Rytov Dip intensity at high temperature $T_{L}$ is connected to the intensity of both transverse phonon peaks at low temperature $T_{G}$ via

$$
\begin{aligned}
& I^{\operatorname{Dip}}\left(T_{L}\right) / I_{\mathrm{ph}}^{H V}\left(T_{G}\right) \\
& \quad=\frac{T_{L}}{T_{G}}\left[\frac{T_{\infty}\left(T_{L}\right)}{T_{\infty}\left(T_{G}\right)}\right]^{2}\left[q c_{t}\left(T_{G}\right) \tau_{T}\left(T_{L}\right)\right]^{2} .
\end{aligned}
$$

This indicates, whether the Rytov Dip is observable, if the glass spectrum is known.

\section{MODE COUPLING THEORY}

In the previous chapters microscopic formulas for the light scattering spectra of undercooled liquids were derived. The starting point was a well known dielectric model. ${ }^{13}$ The expressions above contain four-point correlation functions which cannot be calculated exactly. A standard approximation applied to such correlators is the mode coupling approximation first introduced by Kawasaki. ${ }^{26}$ In general there is no possibility to estimate the quantitative accuracy of this approximation. Applied to fluctuation functions with the full dynamics $R(z)$ it miscalculates the contributions of the hydrodynamic poles. ${ }^{27,23}$ For microscopic frequencies quantitative studies of this approximation for the reduced dynamics $R^{\prime}(z)$ also show serious discrepancies from experiment. The analysis of Ref. 27 of light scattering from liquid helium is critically discussed by Madden. ${ }^{16}$ He points out, that the factorization approximation fails at frequencies $\omega \approx 60 \mathrm{~cm}^{-1} \approx 1 / t_{0}$, which he identifies as microscopic atomic vibrations.

Recently a Mode Coupling Theory (MCT) for the liquid to glass transition has been developed. ${ }^{28,29}$ It factorizes the density pair fluctuation function only after projecting out the hydrodynamic poles and concentrates on the slow relaxation processes in a supercooled liquid for $\omega t_{0} \ll 1$. The theoretical predictions for slow relaxation processes in a hard sphere system at the glass transition are in quantitative agreement with experiments on colloidal systems. ${ }^{30,31}$ Even in more complex systems specific predictions of the MCT have been checked experimentally, for example. ${ }^{32-35}$ Only such general predictions of the theory and their implications for light scattering spectra shall be quoted in this paper. The emphasis here is on the reduction of the dielectric fluctuations to density fluctuations. For the development of the MCT and for proofs the reader is referred to recent reviews. ${ }^{36,37}$

The MCT predicts a glass transition temperature $T_{c}$ below which the idealized $\alpha$ peak is frozen, i.e., $\tau \rightarrow \infty$ and

$$
\Phi_{q}(t \rightarrow \infty) \rightarrow f_{q}>0 \text { or } \phi_{q}(z \rightarrow 0) \rightarrow \frac{-f_{q}}{z} .
$$

$\Phi_{q}(t)$ is the normalized wave vector dependent density correlator $\Phi_{q}(t)=\Phi^{n}(q, t) / \Phi^{n}(q, t=0)$. The existence of the transition temperature $T_{c}$ lying above the calorimetric glass transition temperature was experimentally established in a number of glass forming systems. Among these systems there are molecular dynamics simulations, ${ }^{38,39}$ ionic, ${ }^{40}$ and molecular systems, ${ }^{41,42}$ polymers, ${ }^{43,34,35}$ and the already mentioned colloidal suspensions. ${ }^{30}$ In the last system first principle calculations of the transition temperature using the mode coupling approximation resulted in ten percent deviations from the experimental value. ${ }^{44,28}$ The dynamical anomalies connected with $T_{c}$ are more easily studied in the so called $\beta$ regime $^{45}$ than in the lower lying $\alpha$ relaxation. Moreover the idealized MCT makes few predictions for temperatures below $T_{c}$. The detailed dielectric measurements ${ }^{46}$ of the $\alpha$ relaxation in a large number of materials therefore cannot yet rule out the existence of $T_{c}$ at higher temperatures than studied. The freezing of density fluctuations and of fluctuations of products of densities is stated to be the essence of the glass transition. The dielectric model, which gives $\delta \epsilon$ in terms of density and density pair fluctuations takes up this picture.

Phonon assisted hopping processes brcak the idcalized freezing and restore ergodicity in the glass. ${ }^{47} \mathrm{~A}$ similar mechanism restoring ergodicity was earlier introduced by Mazenko using a fluctuating nonlinear hydrodynamics. ${ }^{48}$ With regard to light scattering experiments the difference in both approaches in the small wave vector regime is important. Below $T_{c}$ the extended MCT leads to a wave vector independent $\alpha$ relaxation time $\tau(q)=$ const. for $q \rightarrow 0 .{ }^{47}$ However the authors of the fluctuating hydrodynamics concluded, 
that the Mountain peak half-width should be proportional to $q^{2}$ for small $q$ if the longitudinal viscosity becomes large. ${ }^{48,49}$ The schematic wave vector independent model studied in Ref. 49 also shows no $\alpha$ relaxation stretching, whereas nongeneric MCT models lead to non-Lorentzian $\alpha$ decay. ${ }^{51}$

In this paper focusing on the qualitative change of light scattering spectra only the idealized theory is presented.

\section{A. Reduction to density fluctuations}

The MCT obtains closed equations for the dynamics of the $\Phi_{q}(t)$ by factorizing the longitudinal memory kernel $K_{l}(t)$ (Ref. 28)

$$
K_{l}(t)=\frac{1}{2} \sum_{k_{p}} V_{l}(q ; k, p) \Phi_{k}(t) \Phi_{p}(t) .
$$

The same approximation ${ }^{28}$ reduces the transverse memory kernel to

$$
K_{s}(t)=\frac{1}{2} \sum_{k p} V_{s}(q ; k, p) \Phi_{k}(t) \Phi_{p}(t) .
$$

The correlation functions $T(t)$ and $\Phi^{F^{\prime} n^{2}}$ can be expressed in terms of the $\Phi_{k}(t)$ applying the same approximations.

Projecting $\left.R^{\prime}(t) L \mid j_{\alpha}(\mathbf{q})\right)$ onto density pairs gives

$$
\begin{aligned}
& \left(n(\mathbf{q}-\mathbf{k}) n(\mathbf{k})\left|R^{\prime}(t) L\right| j_{\alpha}(\mathbf{q})\right) \\
& \quad \approx \sum_{\mathbf{p}} \frac{k T}{m N}\left[\mathbf{q}-\mathbf{p} n c_{\mathbf{p}}-(\mathbf{q}-\mathbf{p}) n c_{\mathbf{q}-\mathbf{p}}\right] \\
& \quad \quad \times\left(n(\mathbf{q}-\mathbf{k}) n(\mathbf{k})\left|R^{\prime}(t)\right| n(\mathbf{q}-\mathbf{p}) n(\mathbf{p})\right),
\end{aligned}
$$

where $c_{k}$ is the direct correlation function; $S_{k}=1 /\left(1-n c_{k}\right)$. The density pair fluctuation function with reduced dynamics $R^{\prime}(t)$ is factorized into a pair of density fluctuations with complete dynamics:

$$
\begin{aligned}
& \left(n(\mathbf{q}-\mathbf{k}) n(\mathbf{k})\left|R^{\prime}(t)\right| n(\mathbf{q}-\mathbf{p}) n(\mathbf{p})\right) \\
& \quad \approx \frac{N^{2}}{k T} S_{k} S_{|\mathbf{q}-\mathbf{k}|} \Phi_{k}(t) \Phi_{|\mathbf{q}-\mathbf{k}|}(t) \delta_{\mathbf{k}, \mathbf{p}},
\end{aligned}
$$

In order to obtain $T^{\mathrm{MCT}}(z)$ the curly bracket in Eq. (41) has to be expanded in $q$. The angle integration in Eq. (9) leaves the following result because of the vanishing trace of $F_{i j}^{\prime}(\mathbf{k})$ :

$$
T^{\mathrm{MCT}}(t)=\frac{1}{15 \pi^{2} n} \int_{0}^{\infty} d k k^{3} \frac{\partial S_{k}}{\partial k} \Phi_{k}^{2}(t) .
$$

The exact result for $T(t=0)$ is recovered because of the normalization $\Phi_{k}(t=0)=1$.

Due to the factorization only $\mathbf{k}^{\prime}=\mathbf{k}$ contributes in the double integral of $\Phi^{F^{\prime} n^{2}}$. The following result is obtained:

$$
\Phi_{\text {MCT }}^{F^{\prime} n^{2}}(t)=\frac{N}{k T} \frac{n}{15 \pi^{2}} \int_{0}^{\infty} d k\left[k S_{k} \Phi_{k}(t)\right]^{2} .
$$

Contrary to the result for $T(t=0)$ Eq. (44) diverges for $t \rightarrow 0$. Assuming the finiteness of $\Phi^{F^{\prime} n^{2}}$ the mentioned restriction $\omega t_{0} \ll 1$ for the factorization approximation is necessary. However dropping the assumption of point particle polarizabilities leads to an effective upper cut off $k_{\max } a \approx 1$ rendering the integral finite. ${ }^{27}$ This generalization of the dielectric model is not considered further.
The light scattering spectra are now expressed in terms of the $\Phi_{k}(t)$. In the preceding chapter the high frequency limits of the $\alpha$ process in different quantities have been introduced. These limits, for example $T_{\infty}$ and $\Phi_{\infty}^{F^{\prime} n^{2}}$, can be obtained from formulas (43) and (44) by substituting $f_{k}$ for $\Phi_{k}(t)$.

\section{B. Dynamics of the density fluctuations}

The MCT predicts an idealized ergodic to nonergodic transition at some critical density $n_{c}$, temperature $T_{c}$ or pressure $p_{c}$. Close to this transition two time scales and processes appear. The already mentioned $\alpha$ process on time scale $\tau$ is the slowest relaxation process and is described only on the liquid side $T>T_{c}$. The $\beta$ process takes place on a mesoscopic time scale $t_{o}$ between $\alpha$ scale $\tau$ and microscopic times denoted by $t_{0}$. In this second region a general correlator of variables of even time inversion symmetry is given by $^{50}$

$$
(X|R(t)| Y)=f_{X Y}^{c}+h_{X Y}^{c} c_{\sigma} g_{ \pm}\left(t / t_{\sigma}\right) \quad \text { for } t_{0} \ll t \ll \tau .
$$

If density fluctuations $\Phi_{q}(t)$ are considered this implies a factorization of space dependence $f_{q}^{c}, h_{q}^{c}$, and time dependence $g_{ \pm}(\hat{t})$. This factorization allows to reduce the wave vector integrals in $K_{l}(t), K_{s}(t), \Phi^{F^{\prime} n^{2}}(t)$, and $T(t)$ to constant prefactors of the interesting dynamics $g_{ \pm}(\hat{t})$.

One exponent parameter $\lambda$ rules the temperature dependence of the time scales and the dynamics on scale $t_{\sigma}{ }^{51}$ It is determined by the microscopic, static equilibrium distribution functions. ${ }^{50}$ How the microscopic structure enters depends strongly on the mode coupling approximation leading to Eq. (40). The value of $\lambda$ obtained for a hard sphere system $^{52}$ could only be varied by four percent in order to describe the $\beta$ dynamics of a hard sphere colloidal suspension. ${ }^{31}$ In complex glass formers $\lambda$ has to be treated as a single fit parameter. $\sigma$ measures the distance to the transition. It also determines the correlation scale $c_{\sigma}=\sqrt{|\sigma|}$. Positive $\sigma$ indicates the glass, where $g_{+}(\hat{t})$ describe the dynamics. The $\alpha$ relaxation time scale diverges as

$$
\tau=t_{0} / \sigma^{\gamma}, \quad \gamma=\frac{1}{2 a}+\frac{1}{2 b},
$$

whereas $t_{v}$ increases according to

$$
t_{\sigma}=t_{0} / \sigma^{1 / 2 a},
$$

$t_{0}$ being insensitive to changes of $\sigma$. The exponents $a$ and $b$, which are given by $\lambda$ via

$$
\begin{aligned}
\lambda=\frac{\Gamma^{2}(1-a)}{\Gamma(1-2 a)}= & \frac{\Gamma^{2}(1+b)}{\Gamma(1+2 b)} ; \\
& 0<a<\frac{1}{2}, 0<b<1
\end{aligned}
$$

also determine the short and long time expansion of $g_{ \pm}(\hat{t})$

$$
\begin{aligned}
& g_{ \pm}(\hat{t}) \rightarrow 1 / \hat{t}^{a} \quad \text { for } \hat{t}=t / t_{\sigma} \ll 1, \\
& g_{+}(\hat{t}) \rightarrow 1 / \sqrt{1-\lambda} \text { for } \hat{t}=t / t_{\sigma} \gg 1,
\end{aligned}
$$

The long time limit in Eqs. (49) together with Eq. (45) leads to a square root behavior of the nonergodicity parameter in 
the glass: $f_{q}=f_{q}^{c}+h_{q}^{c} \sqrt{|\sigma| /(1-\lambda)}$ for $\sigma>0$. In the liquid the following long time limit is found:

$$
g_{-}(\hat{t}) \rightarrow-\hat{B t}^{b} \text { for } \hat{t}=t / t_{\sigma} \gg 1 \text {. }
$$

The von Schweidler law (50) is the essence of the stretching of the $\alpha$ peak, which obeys a scaling law on the liquid side close to $T_{c}$ :

$$
\begin{aligned}
& \lim _{\sigma \rightarrow-0} \Phi_{q}(\tilde{t} \tau)=F_{q}(\tilde{t}), \\
& F_{q}(\tilde{t})=f_{q}^{c}-B h_{q}^{c} \tilde{t}^{b} \text { for } \tilde{t} \ll 1 .
\end{aligned}
$$

The transient dynamics for $\omega t_{0} \approx 1$ and the $\alpha$ relaxation dynamics are not determined by $\lambda$ alonis. Simple MCT models show all generic features observed it the dynamics of real glass formers. In order to generate the figures of this paper the known $F_{12}$ model was used: $:^{51,53}$

$$
\begin{aligned}
& \Phi(z)=-1 /\left[z-\Omega_{0}^{2} /\left(z+i v+\Omega_{0}^{2} m(z)\right)\right], \\
& m(t)=\lambda_{1} \Phi(t)+\lambda_{2} \Phi^{2}(t) .
\end{aligned}
$$

This model claims no connection to a microscopic theory of a real glass former. However its $\beta$ clynamics correctly describes the dynamics of a real system on time scale $t_{\sigma}$ if the exponent parameters $\lambda$ coincide. Thi model also shows the expected $\alpha$ relaxation stretching. The shape of the $\alpha$ peak however depends strongly on the specific polynomial chosen in Eq. (52). ${ }^{54,55}$ The parameters $\Omega_{0}=1 / t_{0}, v=\Omega_{0} / 2$, and $\lambda\left(\lambda_{1}, \lambda_{2}\right)=0.7$ were fixed. $\Phi(t)$ then depends on $\sigma$ alone. Figure 5 shows results for $\sigma=-1 / 80,-0.2,-0.5$ on logarithmic scale $t / t_{0}$. The $\alpha$ relaxation times

$$
\begin{aligned}
& \tau(\sigma=-1 / 80)=10^{5.1} t_{0}, \\
& \tau(\sigma=-0.2)=10^{2.3} t_{0}, \\
& \tau(\sigma=-0.5)=10^{1.2} t_{0},
\end{aligned}
$$

can be taken from the $\alpha$ peaks in the susceptibility $\chi^{\prime \prime}(\omega)=\omega \Phi^{\prime \prime}(\omega)$. From the minimum in $\chi^{\prime \prime}(\omega)$ the $\beta$ scaling time can be estimated to be $t_{\sigma} \approx 10^{3.2} t_{0}$ for $\sigma=-1 / 80$.

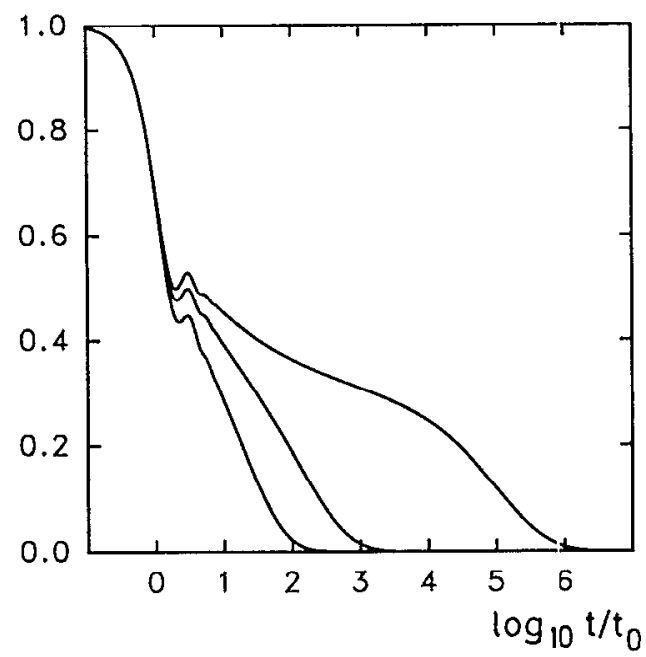

FIG. 5. Correlators $\Phi(t)$ determined by the $F_{12}$ model for the parameters specified in the text. From left to right $\sigma$ takes the values $-0.5,-0.2$, and $-1 / 80$.
Because $\Phi(t)$ has no hydrodynamic contributions it can be taken as input for the fluctuation functions with reduced dynamics $R^{\prime}(t)$. To generate Figs. $1-4$ consistently with the discussion of Sec. IV, the memory functions were approximated by

$$
\begin{aligned}
& K_{s}(t)=\left(G_{\infty} / f\right) \Phi(t), \\
& K_{t}(t)=\frac{M_{\infty}-M_{0}}{f} \Phi(t), \\
& T(t)=T_{0} \Phi(t), \\
& \Phi^{F^{\prime} n^{2}}(t)=\frac{N}{k T} n^{2} S_{q} \Phi_{0}^{F^{\prime} n^{2}} \Phi(t) .
\end{aligned}
$$

Within the $F_{12}$ model $f=1-\lambda=0.3$ is already fixed. Let us emphasize again that ansatz (54) in general describes the dynamics only qualitatively. For example it fixes the ratios of $h_{X Y}^{c} / f_{X Y}^{c}$ to the same artificial value in the four functions Eq. (54). The ansatz however satisfies, that in the mesoscopic time range on scale $t_{\sigma}$ the dynamics is determined by $g\left(t / t_{\sigma}\right)$ alone.

The experimental time scales were set by $q c_{0} t_{0}=10^{-3}$, $c_{\infty}^{2}=2.43 c_{0}^{2}$ and $c_{t}^{2}=\frac{1}{3} c_{0}^{2}$. for $T_{0}$ the $t=0$ value $T(t=0)=\frac{2}{5}$ was entered.

Typical values of $\alpha n=1$ and $\Phi_{0}^{F^{\prime} n^{2}}=0.1$ lead to Figs. 14, which differ only in their values of $\sigma$ and consecutively $\tau$. From the inclusion of the asymptotic law $\Phi^{\prime \prime}(\omega) \propto 1 / \omega^{1-a}$ with $a=0.327$ the difficulty of extracting the value of $a$ is seen. An unprejudiced least square fit leads to $1-a=0.74 \pm 0.02$.

\section{SUMMARY AND CONCLUSION}

In this paper a model for light scattering was developed, which can explain the change of the spectra from liquid to solid like when going through the glass transition. Except for choosing a simple dielectric model no further approximations are necessary in order to obtain the qualitative behavior. For quantitative comparisons with experiments a realistic dielectric model is required.

The observation of a Rytov Dip for systems of optically isotropic particles is predicted, if the corresponding relaxation times grow large enough. The Rytov Dip is a direct result of the coupling of the transverse current to a traceless tensor, which is proportional to $\delta \epsilon_{i j}$. This result of symmetry considerations - two density fluctuations form an anisotropic unity -is obtained in the low frequency limit of the elastooptic coupling function. The high frequency limit corresponds to the phenomenological ansatz coupling dielectric fluctuations to the strain via the Pockels constants.

Applying the factorization approximation to the exact fluctuation and coupling functions the scattered spectra can be expressed in terms of density fluctuations alone. The necessity of exactly taking into account the hydrodynamic contributions before factorizing is seen in the low frequency Brillouin spectra.

Simple mode coupling models show the stretched dynamics on diverging time scales allowing to describe the qualitative change of the spectra. In a frequency region accessible by light scattering experiments few parameters 
predictions of the MCT can be checked. In principle a calculation of all spectral features is possible within the MCT.

\section{ACKNOWLEDGMENT}

The authors thank Professor W. Götze for helpful discussions and a critical reading of the manuscript.

\section{APPENDIX A}

Using a linear, generalized hydrodynamic formalism fluctuations and correlators of arbitrary quantities are expressed in terms of the conserved hydrodynamic variables density $n$, current $\mathbf{j}$, and temperature $T \cdot{ }^{17}$ Temperature fluctuations are not included in the following short summary. The equations simplify for Laplace transformed quantities: $R(z)=i \int_{0}^{\infty} d t e^{i z t} R(t)$ for $\operatorname{Im} z>0$. The complete Liouvillian dynamics $X(t)=R(t) X(0)=e^{i L t} X(0)$ of a general variable $X(t)$ is changed to the reduced dynamics $R^{\prime}(t)=Q e^{i Q L Q t} Q$ by projecting out the hydrodynamic poles. $Q$ the projection operator satisfies $Q \mid n(\mathbf{q}))=Q \mid \mathbf{j}(\mathbf{q}))=0$. Abbreviating the conserved quantities $n(q)$ and $\mathbf{j}(q)$ as $A_{i}$ and using their orthogonality one obtains the following two formulas: ${ }^{17}$

$$
\begin{aligned}
&\left\langle\delta X^{*}(z)\right\rangle=\sum_{i}\left[\left(X \mid A_{i}\right)-\left(X\left|R^{\prime}(z) L\right| A_{i}\right)\right] \\
& \times \frac{1}{\left(A_{i} \mid A_{i}\right)}\left\langle\delta A_{i}^{*}(z)\right\rangle, \\
&(X|R(z)| Y)=\left(X\left|R^{\prime}(z)\right| Y\right)-\sum_{i j}\left[\left(X \mid A_{i}\right)\right. \\
&\left.-\left(X\left|R^{\prime}(z) L\right| A_{i}\right)\right] \frac{1}{\left(A_{i} \mid A_{i}\right)} \\
& \times\left(A_{i}|R(z)| A_{j}\right) \frac{1}{\left(A_{j} \mid A_{j}\right)} \\
& \times\left[\left(A_{j}\left|L R^{\prime}(z)\right| Y\right)-\left(A_{j} \mid Y\right)\right] .
\end{aligned}
$$

\section{APPENDIX B}

$T(t=0)$ drops out of the formula for the total scattered intensities. At high frequencies $\omega t_{0}>1$ the different terms containing $T(t=0)$ cancel in the spectra. Calculating it however shows, that the mode coupling approximation for it is exact and that a finite value is obtained

$$
\begin{aligned}
T(t=0)= & \lim _{q \rightarrow 0} \frac{3 / 2}{n q_{3}} \int \frac{d^{3} k}{(2 \pi)^{3}}\left(\frac{1}{3}-\hat{k}_{3}^{2}\right) \\
& \times \frac{\left(n(\mathbf{q}-\mathbf{k}) n(\mathbf{k})|Q L| j_{3}(\mathbf{q})\right)}{\left(j_{3}(\mathbf{q}) \mid j_{3}(\mathbf{q})\right)} .
\end{aligned}
$$

Due to the angle integration containing the traceless tensor $F_{i j}^{\prime}(\mathbf{k})$ the projection $Q$ drops out. In linear order in $q$ remains:

$$
\begin{aligned}
\left(n(\mathbf{q}-\mathbf{k}) n(\mathbf{k})|L| j_{\alpha}(\mathbf{q})\right) \doteq & \left(j_{3}(\mathbf{q}) \mid j_{3}(\mathbf{q})\right) \\
& \times\left(q_{\alpha} S_{k}-\frac{\partial S_{k}}{\partial k} \frac{k_{\beta} k_{\alpha}}{k} q_{\beta}\right) .
\end{aligned}
$$

$T(t=0)$ therefore reads

$$
T(t=0)=\frac{1}{15 \pi^{2}} \frac{1}{n} \int_{0}^{\infty} d k k^{3} \frac{\partial S_{k}}{\partial k} .
$$

If the interparticle potential is sufficiently smooth at small distances so that $k^{3}\left(S_{k}-1\right) \rightarrow 0$ is fulfilled for $k \rightarrow \infty$ a partial integration leads to a formula which can be recognized as the Fourier backtransform of the pair distribution function $g(r)$,

$$
\begin{aligned}
T(t=0) & =\frac{-2}{5} \frac{1}{n} \lim _{r \rightarrow 0} \int \frac{d^{3} k}{(2 \pi)^{3}} e^{-i \mathbf{k r}}\left(S_{k}-1\right) \\
& =\frac{-2}{5} \lim _{r \rightarrow 0}[g(r)-1] .
\end{aligned}
$$

If the general limit $g(0)=0$ is put in, the mentioned result $T(t=0)=\xi \xi$ is obtained.

'B. J. Berne and R. Pecora, Dynamic Light Scattering (Wiley, New York, 1976).

${ }^{2}$ G. D. Patterson and A. Munoz-Rojas, Annu. Rev. Phys. Chem. 38, 191 (1987).

${ }^{3} \mathrm{M}$. Grimsditch and L. M. Torell, in Dynamics of Disordered Materials, edited by D. Richter, A. J. Dianoux, W. Petry, and J. Teixeira (Springer, Berlin, 1989), p. 196

${ }^{4}$ A. Einstein, Ann. Phys. 33, 1275 (1910).

${ }^{5}$ L. D. Landau and E. M. Lifshitz, Elektrodynamik der Kontinua (Akademie, Berlin, 1985).

${ }^{6}$ T. Keyes and D. Kivelson, J. Chem. Phys. 54, 1786 (1971).

${ }^{7}$ H. C. Andersen and R. Pecora, J. Chem. Phys. 54, 2584 (1971).

${ }^{8}$ W. M. Gelbart, Adv. Chem. Phys. 24, 1 (1974).

${ }^{9}$ R. A. MacPhail and D. Kivelson, J. Chem. Phys. 80, 2102 (1984).

${ }^{10} \mathrm{~J}$. Wong and C. A. Angell, Glass: Structure by Spectroscopy (Dekker, Basel, 1976).

${ }^{1}$ N. O. Brige and S. R. Nagel, Phys. Rev. Lett. 54, 2674 (1985).

${ }^{12}$ S. M. Rytov, Zh. Eksp. Teor. Fiz. 33, 514 (1957) [Sov. Phys. JETP 6, 401 (1958)].

${ }^{13}$ B. U. Felderhof, Physica 76, 486 (1974).

${ }^{14}$ G. I. A. Stegeman and B. P. Stoicheff, Phys. Rev. A 7, 1160 (1973).

${ }^{15} \mathrm{G}$. Szamel and J. Piasecki (preprint, 1990).

${ }^{16}$ P. A. Madden, Mol. Phys. 36, 365 (1978).

${ }^{17}$ W. Götze and A. Latz, J. Phys. Condensed Matter. 1, 4169 (1989).

${ }^{18}$ L. D. Landau and E. M. Lifshitz, Theory of Elasticity (Pergamon, London, 1959).

${ }^{19}$ P. A. Madden and D. Kivelson, Adv. Chem. Phys. 64, 467 (1984).

${ }^{20}$ P. A. Madden and K. O'Sullivan, J. Phys. Condensed Matter 2, SA 257 (1990).

${ }^{21}$ D. Forster, Hydrodynamic Fluctuations, Broken Symmetry, and Correlation Functions (Benjamin, Reading, 1975).

${ }^{22}$ J. P. Boon and S. Yip, Molecular Hydrodynamics (Wiley, New York, 1980).

${ }^{23}$ N. J. Tao, G. Li, X. Chen, and H. Z. Cummins (preprint, 1990).

${ }^{24}$ W. S. Gornall, H. E. Howard-Lock, and B. P. Stoicheff, Phys. Rev. A 1, 1288 (1970).

${ }^{25}$ R. D. Mountain, J. Res. Natl. Bur. Stand. Sect. A 70, 207 (1966).

${ }^{26}$ K. Kawasaki, Phys. Rev, 150, 291 (1966).

${ }^{27}$ M. J. Stephen, Phys. Rev, 187, 247 (1969).

${ }^{28}$ U. Bengtzelius, W. Götze, and A. Sjölander, J. Phys. C 17, 5915 (1984).

${ }^{29}$ E. Leutheuser, Phys. Rev. A 29, 2765 (1984).

${ }^{30}$ W. van Megen and P. N. Pusey, Phys. Rev. A 43, 5429 (1991).

${ }^{3}$ 'W. Götze and L. Sjögren, Phys. Rev. A 43, 5442 (1991).

${ }^{32}$ F. Mezei, W. Knaak, and B. Farago, Phys. Rev. Lett. 58, 571 (1987).

${ }^{33}$ W. Doster, S. Cusack, and W. Petry, Phys. Rev. Lett. 65, 1080 (1990).

${ }^{34} \mathrm{~L}$. Sjögren, in Basic Features of the Glassy State, edited by J. Colmenero and A. Alegria (World Scientific, Singapore, 1990), p. 137.

${ }^{35}$ L. Sjögren, J. Phys. Condensed Matter 3, 5023 (1991).

${ }^{36} \mathrm{~W}$. Götze, in Liquids Freezing and the Glass Transition, edited by D. Levesque, J. P. Hansen, and J. Zinn-Justin (Elsevier, New York, 1991).

${ }^{37} \mathrm{~L}$. Sjögren and W. Götze, in Dynamics of Disordered Materials, edited by D. Richter, A. J. Dianoux, W. Petry, and J. Teixeira (Springer, Berlin, 1989), p. 18. 
${ }^{38}$ J. N. Roux, J. L. Barrat, and J. P. Hansen, J. Phys. Condensed Matter 1, 7171 (1989); J. L. Barrat, J. L. Roux, and J. P. Hansen, Chem. Phys. 149, 197 (1990).

${ }^{39}$ G. F. Signorini, J. L. Barrat, and M. L. Klein, J. Chem. Phys. 92, 1294 (1990).

${ }^{10} \mathrm{~F}$. Mezei, Phys. Scr. T 19, 363 (1987); Dynamics of Disordered Materials, edited by D. Richter, A. J. Dianoux, W. Petry, and J. Teixeira (Springer, Berlin, 1989), p. 164.

A'E. Bartsch, M. Kiebel, H. Sillescu, and W. Petry, Ber. Bunsenges Phys. Chem. 93, 1252 (1989); W. Petry, E. Bartsch, F. Fujara, M. Kiebel, H. Sillescu, and B. Farago, Z. Phys. B 83, 175 (1991).

${ }^{42}$ L. Börjesson, M. Elmroth, and L. Torell, Chem. Phys. 149, 209 (1990).

${ }^{43}$ B. Frick, D. Richter, W. Petry, and U. Buchenau, Z. Phys. B 70, 73 (1988); B. Frick, B. Farago, and D. Richter, Phys. Rev. Lett. 64, 2921 (1990).

${ }^{44}$ P. Pusey and W. van Megen, Phys. Rev. Lett. 59, 2083 (1987).
${ }^{45}$ N. J. Tao, G. Li, and H. Z. Cummins, Phys. Rev. Lett. 66, 1334 (1991). ${ }^{46}$ P. K. Dixon, L. Wu, S. R. Nagel, B. D. Williams, and J. P. Carini, Phys. Rev. Lett. 65, 1108 (1990).

${ }^{47}$ W. Götze and L. Sjögren, Z. Phys. B 65, 415 (1987).

${ }^{48}$ S. P. Das and G. F. Mazenko, Phys. Rev. A 34, 2265 (1986).

${ }^{49}$ B. Kim and G. F. Mazenko, Adv. Chem. Phys. 78, 129 (1991).

${ }^{50}$ W. Götze, Z. Phys. B 60, 195 (1985).

${ }^{51}$ W. Götze, Z. Phys. B 56, 139 (1984).

${ }^{52}$ J. L. Barrat, W. Götze, and A. Latz, J. Phys. Condensed Matter 1, 7163 (1989).

${ }^{53}$ W. Götze and L. Sjögren, J. Phys. C 21, 3407 (1988).

${ }^{54}$ G. Buchalla, U. Dersch, W. Götze, and L. Sjögren, J. Phys. C 21, 4239 (1988).

${ }^{55}$ M. Fuchs, W. Götze, I. Hofacker, and A. Latz, J. Phys. Condensed Matter 3, 5047 (1991). 